ALEA, Lat. Am. J. Probab. Math. Stat. 13, 1165-1187 (2016)

DOI: 10.30757/ALEA.v13-42

\title{
On the rate of convergence in de Finetti's representation theorem
}

\section{Guillaume Mijoule, Giovanni Peccati and Yvik Swan}

Université de Liège.

Département de Mathématique - zone polytech 1

12 allée de la découverte Bât. B37 pkg 33a B-4000 Liège

E-mail address: guillaume.mijoule@gmail.com

Université du Luxembourg

E-mail address: giovanni.peccati@gmail.com

Université de Liège.

Département de Mathématique - zone polytech 1

12 allée de la découverte Bât. B37 pkg 33a B-4000 Liège

E-mail address: yswan@ulg.ac.be

\begin{abstract}
A consequence of de Finetti's representation theorem is that for every infinite sequence of exchangeable 0-1 random variables $\left(X_{k}\right)_{k \geq 1}$, there exists a probability measure $\mu$ on the Borel sets of $[0,1]$ such that $\bar{X}_{n}=n^{-1} \sum_{i=1}^{n} X_{i}$ converges weakly to $\mu$. For a wide class of probability measures $\mu$ having smooth density on $(0,1)$, we give bounds of order $1 / n$ with explicit constants for the Wasserstein distance between the law of $\bar{X}_{n}$ and $\mu$. This extends a recent result by Goldstein and Reinert (2013) regarding the distance between the scaled number of white balls drawn in a Pólya-Eggenberger urn and its limiting distribution. We prove also that, in the most general cases, the distance between the law of $\bar{X}_{n}$ and $\mu$ is bounded below by $1 / n$ and above by $1 / \sqrt{n}$ (up to some multiplicative constants). For every $\delta \in[1 / 2,1]$, we give an example of an exchangeable sequence such that this distance is of order $1 / n^{\delta}$.
\end{abstract}

\section{Introduction}

1.1. Overview and framework. An infinite sequence $\left(X_{k}\right)_{k \geq 1}$ of random variables is exchangeable if for every $n \geq 1$ and every permutation $\sigma$ of $\{1, \ldots, n\}$, $\left(X_{\sigma(1)}, \ldots, X_{\sigma(n)}\right)$ has the same distribution as $\left(X_{1}, \ldots, X_{n}\right)$. The following fundamental theorem was discovered by De Finetti $(1929,1930,1980)$ :

Received by the editors January 25, 2016; accepted November 27, 2016.

2010 Mathematics Subject Classification. 60F05, 60G09.

Key words and phrases. de Finetti's theorem, Exchangeable Variables, Wasserstein distance, Urn models. 
Theorem 1.1. (de Finetti, 1937) An infinite sequence $\left(X_{k}\right)_{k>1}$ of 0-1 random variables is exchangeable if, and only if, there exists a (necessarily unique) probability measure $\mu$ on the Borel sets of $[0,1]$ such that for every $n \geq 1$ and every $\left(e_{1}, \ldots, e_{n}\right) \in$ $\{0,1\}^{n}$,

$$
\mathbb{P}\left[X_{1}=e_{1}, \ldots, X_{n}=e_{n}\right]=\int_{0}^{1} t^{k}(1-t)^{n-k} \mu(d t),
$$

where $k=\sum_{i=1}^{n} e_{i}$.

Exchangeability has been extensively studied in the literature. Hewitt and Savage (1955) extend de Finetti's result for variables taking values in general spaces. Diaconis and Freedman (1980) give an approximation result when the sequence $\left(X_{k}\right)_{k \geq 1}$ is finite, in which case, a representation of the type (1.1) does not necessarily hold. For an overview of results related to exchangeability, we refer to the classical lecture notes Aldous (1985), as well as to Austin (2008); Kallenberg (2005); Pitman (1996) for more recent accounts.

Equation (1.1) has an elegant Bayesian interpretation, namely: the law of $\left(X_{k}\right)_{k \geq 1}$ is that of a sequence of i.i.d. Bernoulli random variables with parameter $\theta$ randomly chosen from the (prior) probability measure $\mu$. The measure $\mu$ is sometimes called de Finetti's measure or mixing measure associated with the sequence.

Defining $\bar{X}_{n}=n^{-1} \sum_{i=1}^{n} X_{i}$, we readily obtain the following De Finetti-type Law of Large Numbers (LLN) in distribution:

$$
\bar{X}_{n} \stackrel{\mathcal{L}}{\longrightarrow} \mu,
$$

where $\mathcal{L}$ indicates weak convergence. Relation (1.2) is easy to see with the Bayesian point of view of (1.1) : if, on some probability space, we are given a random variable $\theta$ with distribution $\mu$ and a sequence $\left(X_{k}\right)_{k \geq 1}$ which are, conditionally on $\theta$, Bernoulli i.i.d. random variables with parameter $\theta$, then $\bar{X}_{n}$ converges almost surely to $\theta$. Hence, convergence in distribution also holds.

Conditions under which LLNs for exchangeable sequences hold have, naturally, been extensively studied in the literature see Hanson and Koopmans (1965); Inoue and Taylor (2006); Stoica (2011); Taylor and Hu (1987) or the more general Taylor et al. (1985). There has however been only little investigation into explicit rates of convergence for the distributional limit theorem in (1.2) for general mixture measures $\mu$. One of the results of Hauray and Mischler (2014) is a bound of the order $1 / \sqrt{n}$ whenever the $X_{k}$ take values in a subspace of $\mathbb{R}^{d}$; however, in our much simpler framework where $X_{k}$ is 0 or 1 , such a bound is not hard to obtain directly (see Proposition 3.1). We also mention that, although the main result of Diaconis and Freedman (1980) is sometimes refered to as quantitative de Finetti theorem (or quantum de Finetti theorem), it is a completely different problem that we investigate here: in Diaconis and Freedman (1980), the exchangeable sequence is supposed to be finite, and a bound is obtained for the distance between the distribution of $\left(X_{1}, \ldots, X_{n}\right)$ and the set of mixture measures of the type (1.1). Of course, if the sequence is infinite (which is what we assume), de Finetti's theorem exactly says that this distance is zero.

Another line of research deals with a possible central limit theorem for exchangeable sequences, that is, the search of conditions under which $\sum_{i=1}^{n} X_{i}$ converges towards a normal distribution (once centered and properly renormalized). The seminal paper in this direction is due to Blum et al. (1958), where conditions are given for $n^{-1 / 2} \sum_{i=1}^{n} X_{i}$ to be asymptotically Gaussian. In our framework of $0-1$ 
random variables, this happens only in the i.i.d case. More recent developments can be found in Klass and Teicher (1987); Weber (1980).

To the best of our knowledge, the closest result to ours is due to Goldstein and Reinert (2013) and concerns the classical Pólya-Eggenberger urn model (see Section 4.2 for a description of the model, and see e.g. Pitman (1996) for a general discussion, as well as El-Dakkak and Peccati (2008); El-Dakkak et al. (2014); Peccati (2004) for several recent developments). In this case, the mixing measure is a Beta distribution. One of the results of Goldstein and Reinert (2013) is that the Wasserstein distance between the scaled number of drawn white balls and its corresponding limiting Beta distribution is of order $1 / n$. The proof is based on a version of Stein's method as adapted to the Beta distribution. We also mention a similar bound obtained by Döbler (2015).

We recall that the Wasserstein distance between the distributions of two realvalued and integrable random variables $X, Y$ is given by the quantity

$$
d_{W}(X, Y)=\sup _{\phi \in \operatorname{Lip}(1)}|\mathbb{E}[\phi(X)]-\mathbb{E}[\phi(Y)]|,
$$

where $\operatorname{Lip}(K)$ is the set of $K$-Lipschitz functions on the real-line. It is a well-known (and easily checked) fact that the topology induced by $d_{W}$ on the class of probability measures on the real line is strictly stronger than the topology of convergence in distribution. In the framework of the present paper, it is also interesting to notice that, if one restricts oneself to the collection of all probability measures supported on $[0,1]$ then the two topologies are actually equivalent (to see this one can e.g. use the representation (2.3) below, and then exploit the Dominated Convergence theorem).

It is the goal of this paper to estimate the rate of convergence in Wasserstein distance for the distributional limit theorem in (1.2).

1.2. Main results. In this paper, we prove that a bound of the same order as in Goldstein and Reinert (2013) for the Beta target still holds for more general distributions $\mu$. Our main theorem is the following:

Theorem 1.2. Let $\left(X_{k}\right)_{k \geq 1}$ be an infinite sequence of 0-1 exchangeable variables, $\bar{X}_{n}=n^{-1} \sum_{i=1}^{n} X_{i}$ and $\mu$ the limiting distribution of $\bar{X}_{n}$. Suppose $\mu$ has a smooth density $p$ on $(0,1)$ satisfying

$$
\int_{0}^{1} u(1-u)\left|p^{\prime}(u)\right| d u<\infty .
$$

Then with $\theta \sim \mu$,

$$
\frac{C_{1}}{n} \leq d_{W}\left(\bar{X}_{n}, \theta\right) \leq \frac{C_{2}}{n},
$$

where $C_{1}$ and $C_{2}$ only depend on $\mu$ and are given by

$$
\begin{aligned}
& C_{1}(\mu)=\int_{0}^{1} u(1-u) p(u) d u, \\
& C_{2}(\mu)=\int_{0}^{1}\left(|1-2 u|+u^{2}+(1-u)^{2}\right) p(u) d u+\int_{0}^{1} u(1-u)\left|p^{\prime}(u)\right| d u+\frac{3}{\sqrt{2 \pi e}} .
\end{aligned}
$$

Remark 1.1. The natural space where the density $p$ satisfying the integrability property (1.4) lives, is the weighted Sobolev space $\mathcal{W}=W_{1,1}((0,1), u \mapsto u(1-u))$, 
defined by those functions $f$ such that $f$ has a weak derivative $f^{\prime}$, and $f$ and $x(1-x) f^{\prime}$ are integrable. It is equipped with the norm

$$
\|f\|_{\mathcal{W}}=\int_{(0,1)}|f(x)| d x+\int_{(0,1)} x(1-x)\left|f^{\prime}(x)\right| d x .
$$

By a density argument, one can generalize the result of Theorem 1.2 to all probability density functions $p \in \mathcal{W}$. For the sake of conciseness, we give here only a sketch of the proof and leave to the interested reader to fill in the details. Note that the second inequality in (1.5) can be rewritten in terms of $p$, by a conditioning argument and $(2.3)$ :

$$
\begin{aligned}
& n \int_{0}^{1}\left|\int_{0}^{1}\left(P_{n, u}(n x)-\mathbf{1}_{u \leq x}\right) p(u) d u\right| d x \\
& \leq \int_{0}^{1}\left(|1-2 u|+u^{2}+(1-u)^{2}\right) p(u) d u+\int_{0}^{1} u(1-u)\left|p^{\prime}(u)\right| d u+\frac{3}{\sqrt{2 \pi e}}
\end{aligned}
$$

where $P_{n, u}$ is the cdf of a Binomial random variable with parameters $n \in \mathbb{N}$ and $u \in[0,1]$. Theorem 1.2 tells us that $(1.7)$ holds for a smooth pdf $p \in \mathcal{W}$. First, one proves that (1.7) holds for a smooth $p \in \mathcal{W}$ (not necessarily a pdf). Then, the proof follows using the denseness of $\mathcal{C}^{\infty}([0,1])$ in $\mathcal{W}$ (e.g. from Theorem 11.2 of Kufner, $1985)$ and the continuity of both sides of (1.7) as functions of $p \in \mathcal{W}$.

Theorem 1.2 will be applied to the case of the Beta distribution, leading to a bound in Wasserstein distance in the Pólya-Eggenberg urn model as explained in Section 4.2 (see Corollary 4.4). We will numerically compare the constants we obtain with the constants in Goldstein and Reinert (2013); it turns out that our result leads to better constants for the wide range of parameter values we investigated.

In view of the above mentioned LLN, it is not hard to prove (see Proposition 3.1) that, whatever the distribution $\mu$ of $\theta$,

$$
\frac{\mathbb{E}[\theta(1-\theta)]}{n} \leq d_{W}\left(\bar{X}_{n}, \theta\right) \leq \sqrt{\frac{\mathbb{E}[\theta(1-\theta)]}{n}} .
$$

Another contribution of this paper is that we prove that such bounds are sharp in the sense that, for every $\delta \in[1 / 2,1]$, we exhibit a measure $\mu$ which violates Assumption (1.4) and such that $d_{W}\left(\bar{X}_{n}, \theta\right)$ is of order $1 / n^{\delta}$; see Proposition 5.3.

The weight function $u \mapsto u(1-u)$ in the integrability property (1.4) appears naturally as the variance of a Bernoulli random variable of parameter $u$; see the proofs of Propositions 3.3 and 4.1. Thus, in statistical terms, the previous Theorem can be stated as follows. For a non-random $\theta, \bar{X}_{n}$ is the maximum likelihood estimator of $\theta$. It is asymptotically normal and has variance $I(\theta)^{-1} / n, I(\theta)$ being the Fisher information of the model. If now $\theta$ is drawn at random from a density $p$, and if $\rho(x)=p^{\prime}(x) / p(x)$ is the score of $p$, then the optimal rate of convergence $\mathcal{O}\left(n^{-1}\right)$ of $d_{W}\left(\bar{X}_{n}, \theta\right)$ is attained if

$$
\mathbb{E}\left[I(\theta)^{-1}|\rho(\theta)|\right]<\infty .
$$

Even though it goes beyond the scope of this paper, we believe it would be interesting to know whether this result holds true in more general Bayesian models.

Let us now briefly sketch our strategy. We know from the classical central limit theorem that, conditionally on $\theta, \sqrt{n}\left(\bar{X}_{n}-\theta\right)$ converges weakly to a normal distribution with variance $\theta(1-\theta)$ (the variance of a Bernoulli variable of parameter $\theta$ ). Moreover, a Berry-Esséen type theorem gives a bound of the Wasserstein distance 
between those two variables. This will allow us to prove (see Proposition 3.3) that controlling $d_{W}\left(\bar{X}_{n}, \theta\right)$ is equivalent (in a sense which will be made precise later on) to controlling $d_{W}\left(\theta+\sqrt{\frac{\theta(1-\theta)}{n}} Z, \theta\right)$ where $Z$ stands for a standard normal random variable independent of $\theta$. Finally, we bound the latter quantity by a purely analytical and elementary (though computationally heavy) method, using the representation of the Wasserstein distance as the $L^{1}$ norm of the difference of the cumulative distribution functions.

The paper is organized as follows. Section 2 gives the basic definitions and notations. In Section 3, we study the trivial cases and we show that the distance between $d_{W}\left(\bar{X}_{n}, \theta\right)$ and $d_{W}\left(\theta+\sqrt{\frac{\theta(1-\theta)}{n}} Z, \theta\right)$ is bounded by $\mathcal{O}(1 / n)$. In Section 4 we bound $d_{W}\left(\theta+\sqrt{\frac{\theta(1-\theta)}{n}} Z, \theta\right)$ from above for regular enough measures $\mu$ and prove Theorem 1.2. Finally, in Section 5 , for every $\delta \in[1 / 2,1]$, we give an example of a measure $\mu$ violating our assumptions and for which the rate of convergence is exactly of order $1 / n^{\delta}$.

\section{Definitions and notations}

Let $\mu$ and $\nu$ be two probability measures on $\mathbb{R}$. The Wasserstein (or Kantorovitch) distance between $\mu$ and $\nu$ is defined as

$$
d_{W}(\mu, \nu)=\inf _{\pi} \iint|x-y| \pi(d x, d y)
$$

where the infimum is taken over all probability measures $\pi$ on $\mathbb{R} \times \mathbb{R}$ with first marginal $\mu$ and second marginal $\nu$. When $X$ and $Y$ are integrable real-valued random variables, $d_{W}(X, Y)$ will denote the Wasserstein distance between the probability measures induced by $X$ and $Y$ on the Borel sets of $\mathbb{R}$. In this case equation (2.1) becomes

$$
d_{W}(X, Y)=\inf \mathbb{E}\left[\left|X^{\prime}-Y^{\prime}\right|\right]
$$

the infimum being taken over all couples of real-valued random variables $\left(X^{\prime}, Y^{\prime}\right)$ such that $X^{\prime}$ (resp. $Y^{\prime}$ ) has the law of $X$ (resp. $Y$ ). From the Kantorovitch duality theorem, we readily deduce the representation (1.3) mentioned in the Introduction. Yet another representation of the Wasserstein distance is given by the $L^{1}$-norm of the difference between the cumulative distribution functions :

$$
d_{W}(X, Y)=\int_{\mathbb{R}}|\mathbb{P}[X \leq x]-\mathbb{P}[Y \leq x]| d x .
$$

For a proof of these equivalent definitions, one can consult e.g. Vallender (1974); Gibbs and Su (2002) or Villani (2003).

At this point, it is worth mentioning the following two standard facts concerning the relation between the Wasserstein distance $d_{W}(X, Y)$ and the so-called Kolmogorov distance

$$
d_{K}(X, Y):=\sup _{x \in \mathbb{R}}|\mathbb{P}[X \leq x]-\mathbb{P}[Y \leq x]| d x
$$

(a) If $Y$ has a density bounded by some constant $A \in(0, \infty)$ and $X$ is integrable, then one has that

$$
d_{K}(X, Y) \leq C \sqrt{d_{W}(X, Y)}
$$


where $C$ is a constant possibly depending on $A$ (one can obtain such an estimate e.g. by mimicking the proof of Chen et al., 2011, Theorem 3.3).

(b) If $X$ and $Y$ take values in $[0,1]$, then

$$
d_{W}(X, Y)=\int_{[0,1]}|\mathbb{P}[X \leq x]-\mathbb{P}[Y \leq x]| d x \leq d_{K}(X, Y) .
$$

In particular, the estimates appearing in (a) and (b) may be combined with Theorem 1.2 , in order to deduce (arguably not optimal) upper and lower bounds on the rate of convergence in the Kolmogorov distance for the limit theorem in (1.2).

Since the quantities of interest here only involve the distribution of the considered random variables, we make the following assumption in the rest of the paper : on some probability space $(\Omega, \mathcal{F}, \mathbb{P})$, we are given a random variable $\theta$ with values in $[0,1]$ and with law denoted $\mu$, and a sequence $\left(X_{k}\right)_{k \geq 1}$ of $0-1$ random variables such that $\left(X_{k}\right)_{k \geq 1}$ are, conditionally on $\theta$, i.i.d. Bernoulli random variables with parameter $\theta$. The distribution of $\left(X_{k}\right)_{k \geq 1}$ is then given by (1.1).

For a sequence of random variables $\left(V_{k}\right)_{k \geq 1}$, we write

$$
\bar{V}_{n}=n^{-1} \sum_{k=1}^{n} V_{k}
$$

We also adopt the following notation : for two real-valued non-negative sequences $\left(a_{n}\right)_{n \geq 1}$ and $\left(b_{n}\right)_{n \geq 1}$, we write $a_{n} \cong b_{n}$ if both $a_{n}=\mathcal{O}\left(b_{n}\right)$ and $b_{n}=\mathcal{O}\left(a_{n}\right)$.

\section{Bounds in the general case}

3.1. Preliminaries. We start with the following simple proposition, which shows that $d_{W}\left(\bar{X}_{n}, \theta\right)$ is bounded from above and below, respectively, by terms of the order $1 / \sqrt{n}$ and $1 / n$.

Proposition 3.1. It holds that

$$
\frac{\mathbb{E}[\theta(1-\theta)]}{n} \leq d_{W}\left(\bar{X}_{n}, \theta\right) \leq \sqrt{\frac{\mathbb{E}[\theta(1-\theta)]}{n}} .
$$

Proof: The Cauchy-Schwarz inequality implies

$$
\begin{aligned}
\mathbb{E}\left[\left|\bar{X}_{n}-\theta\right|\right] & \leq \sqrt{\mathbb{E}\left[\left(\bar{X}_{n}-\theta\right)^{2}\right]} \\
& =\sqrt{\mathbb{E}\left[\mathbb{E}\left[\left(\bar{X}_{n}-\theta\right)^{2} \mid \theta\right]\right]} \\
& =\sqrt{\frac{\mathbb{E}[\theta(1-\theta)]}{n}},
\end{aligned}
$$

giving the upper bound.

To show the lower bound, we use the dual formulation of the Wasserstein distance. First we remark that the function $\psi: x \mapsto x(x-1) \mathbf{1}_{[0,1]}(x)$ is 1-Lipschitz. We have

$$
\begin{aligned}
\mathbb{E}\left[\psi\left(\bar{X}_{n}\right)\right]=\mathbb{E}\left[\bar{X}_{n}\left(\bar{X}_{n}-1\right)\right] & =\mathbb{E}\left[\bar{X}_{n}^{2}\right]-\mathbb{E}\left[\bar{X}_{n}\right] \\
& =\mathbb{E}\left[\mathbb{E}\left[\bar{X}_{n}^{2} \mid \theta\right]\right]-\mathbb{E}[\theta] \\
& =\mathbb{E}\left[\frac{\theta(1-\theta)}{n}+\theta^{2}\right]-\mathbb{E}[\theta] .
\end{aligned}
$$


Thus,

$$
\mathbb{E}\left[\psi\left(\bar{X}_{n}\right)\right]-\mathbb{E}[\psi(\theta)]=\frac{\mathbb{E}[\theta(1-\theta)]}{n} .
$$

From (1.3), we get the desired result.

From the previous Proposition, if $\mu(\{0,1\})=1$ (or, equivalently, $\theta=0$ or $\theta=1$ almost surely), then

$$
d_{W}\left(\bar{X}_{n}, \theta\right)=0 .
$$

Our next lemma shows that, if $\mu(\{0,1\})<1$, then the rate of convergence of $d_{W}\left(\bar{X}_{n}, \theta\right)$ does not change if we "kill" the mass of $\mu$ on $\{0,1\}$.

Lemma 3.2. Assume that $\mu(\{0,1\})<1$. Let $\tilde{\theta}$ have the law $\tilde{\mu}$ defined by $\tilde{\mu}(A)=$ $\mu(A \backslash\{0,1\}) /\left(1-\mu(\{0,1\})\right.$ for all Borel sets $A$ of $\mathbb{R}$, and $\left(Y_{k}\right)_{k \geq 1}$ be a Bernoulli sequence with prior $\tilde{\mu}$. Then $\tilde{\mu}(\{0,1\})=0$ and

$$
d_{W}\left(\bar{X}_{n}, \theta\right)=\left(1-\mu(\{0,1\}) d_{W}\left(\bar{Y}_{n}, \tilde{\theta}\right) .\right.
$$

In particular,

$$
d_{W}\left(\bar{X}_{n}, \theta\right) \cong d_{W}\left(\bar{Y}_{n}, \tilde{\theta}\right) .
$$

Proof: Let $\psi \in \operatorname{Lip}(1)$. Then

$$
\begin{aligned}
\mathbb{E}\left[\psi\left(\bar{X}_{n}\right)\right]-\mathbb{E}[\psi(\theta)] & =\mathbb{E}\left[\psi\left(\bar{X}_{n}\right) \mathbf{1}_{\{\theta=0\}}\right]-\mathbb{E}\left[\psi(\theta) \mathbf{1}_{\{\theta=0\}}\right] \\
& +\mathbb{E}\left[\psi\left(\bar{X}_{n}\right) \mathbf{1}_{\{\theta=1\}}\right]-\mathbb{E}\left[\psi(\theta) \mathbf{1}_{\{\theta=1\}}\right] \\
& +\mathbb{E}\left[\psi\left(\bar{X}_{n}\right) \mathbf{1}_{\{\theta \neq 0 \text { and } \theta \neq 1\}}\right]-\mathbb{E}\left[\psi(\theta) \mathbf{1}_{\{\theta \neq 0 \text { and } \theta \neq 1\}}\right] \\
& =\mathbb{E}\left[\psi\left(\bar{X}_{n}\right) \mathbf{1}_{\{\theta \neq 0 \text { and } \theta \neq 1\}}\right]-\mathbb{E}\left[\psi(\theta) \mathbf{1}_{\{\theta \neq 0 \text { and } \theta \neq 1\}}\right],
\end{aligned}
$$

since, both on $\{\theta=0\}$ and $\{\theta=1\}, \bar{X}_{n}=\theta$ a.s. However, from the very definition of $\left(Y_{k}\right)_{k \geq 1}$,

$$
\begin{aligned}
\mathbb{E}\left[\psi\left(\bar{X}_{n}\right) \mathbf{1}_{\{\theta \neq 0 \text { and } \theta \neq 1\}}\right] & =\int_{(0,1)} \mathbb{E}\left[\psi\left(\bar{X}_{n} \mid \theta=t\right)\right] \mu(d t) \\
& =\mu((0,1)) \int_{(0,1)} \mathbb{E}\left[\psi\left(\bar{X}_{n}\right) \mid \theta=t\right] \frac{\mu(d t)}{\mu((0,1))} \\
& =(1-\mu(\{0,1\})) \int_{(0,1)} \mathbb{E}\left[\psi\left(\bar{Y}_{n}\right) \mid \theta=t\right] \frac{\mu(d t)}{\mu((0,1))} \\
& =(1-\mu(\{0,1\})) \mathbb{E}\left[\psi\left(\bar{Y}_{n}\right)\right] .
\end{aligned}
$$

A similar argument for $\mathbb{E}\left[\psi(\theta) \mathbf{1}_{\{\theta \neq 0 \text { and } \theta \neq 1\}}\right]$ and taking the supremum over all 1-Lipschitz functions $\psi$ gives the desired result.

From now on, we assume that $\mu(\{0,1\})=0$ (equivalently, $0<\theta<1$ a.s.). Exchangeable sequences such that the associated de Finetti measure has support contained in $(0,1)$ are sometimes called non-deterministic - see e.g. El-Dakkak and Peccati (2008); El-Dakkak et al. (2014); Hill et al. (1987); Peccati (2004). 
3.2. Equivalent formulation with a perturbed version of the prior. In this section, we show that the problem of bounding the Wasserstein distance between $\bar{X}_{n}$ and $\theta$ is equivalent in some sense to bounding the Wasserstein distance between $\theta$ and some perturbed version of $\theta$. Recall that we assume $\mu(\{0,1\})=0$. We will make use of a theorem giving a Berry-Esséen type bound in Wasserstein distance in the classical Central Limit Theorem for Bernoulli random variables (a proof can be found in Chen et al., 2011, Corollary 4.1). We quote it here.

Theorem 3.1. (Chen, 2005) Let $\left(V_{k}\right)_{k \geq 1}$ be a sequence of i.i.d. Bernoulli random variables with parameter $t \in(0,1)$. Let $Y_{k}=(t(1-t))^{-1 / 2}\left(V_{k}-t\right)$ (so that $Y_{k}$ has mean 0 and variance 1$)$, and let $\bar{Y}_{n}=n^{-1} \sum_{k=1}^{n} Y_{k}$. Then

$$
d_{W}\left(\sqrt{n} \bar{Y}_{n}, Z\right) \leq \frac{\mathbb{E}\left[\left|Y_{1}\right|^{3}\right]}{\sqrt{n}}=\frac{t^{2}+(1-t)^{2}}{\sqrt{n t(1-t)}},
$$

where $Z$ stands for a standard normal random variable.

The main result of this section is the following proposition.

Proposition 3.3. Let $Z$ stand for a standard normal random variable independent of $\theta$. Then

$$
\left|d_{W}\left(\bar{X}_{n}, \theta\right)-d_{W}\left(\theta+\sqrt{\frac{\theta(1-\theta)}{n}} Z, \theta\right)\right| \leq \frac{\mathbb{E}\left[\theta^{2}+(1-\theta)^{2}\right]}{n} .
$$

Proof: Let $\psi \in \operatorname{Lip}(1)$. For every $t \in(0,1)$, we define the function $\phi_{t}$ by $\phi_{t}(\sqrt{n}(t(1-$ $\left.t))^{-1 / 2}(x-t)\right)=\psi(x)$, or equivalently,

$$
\phi_{t}(x)=\psi\left(t+\sqrt{\frac{t(1-t)}{n} x}\right)
$$

Clearly we have $\phi_{t} \in \operatorname{Lip}\left(\sqrt{\frac{t(1-t)}{n}}\right)$.

Let $Y_{i}=(\theta(1-\theta))^{-1 / 2}\left(X_{i}-\theta\right)$. We have

$$
\begin{aligned}
& \mathbb{E}\left[\psi\left(\bar{X}_{n}\right)\right]-\mathbb{E}[\psi(\theta)] \\
& =\mathbb{E}\left[\mathbb{E}\left[\psi\left(\bar{X}_{n}\right) \mid \theta\right]\right]-\mathbb{E}[\psi(\theta)] \\
& =\mathbb{E}\left[\mathbb{E}\left[\phi_{\theta}\left(\sqrt{n}(\theta(1-\theta))^{-1 / 2}\left(\bar{X}_{n}-\theta\right)\right) \mid \theta\right]\right]-\mathbb{E}[\psi(\theta)] \\
& =\mathbb{E}\left[\mathbb{E}\left[\phi_{\theta}\left(\sqrt{n} \bar{Y}_{n}\right) \mid \theta\right]\right]-\mathbb{E}[\psi(\theta)] .
\end{aligned}
$$

Let $Z$ be a standard normal variable independent of $\theta$. Theorem 3.1 together with the fact that $\phi_{t} \in \operatorname{Lip}\left(\sqrt{\frac{t(1-t)}{n}}\right)$ implies

$$
\begin{aligned}
\mathbb{E}\left[\phi_{\theta}\left(\sqrt{n} \bar{Y}_{n}\right) \mid \theta\right] & \leq \mathbb{E}\left[\phi_{\theta}(Z) \mid \theta\right]+\frac{\theta^{2}+(1-\theta)^{2}}{\sqrt{n \theta(1-\theta)}} \cdot \sqrt{\frac{\theta(1-\theta)}{n}} \\
& =\mathbb{E}\left[\phi_{\theta}(Z) \mid \theta\right]+\frac{\theta^{2}+(1-\theta)^{2}}{n} .
\end{aligned}
$$

Thus,

$$
\mathbb{E}\left[\psi\left(\bar{X}_{n}\right)\right]-\mathbb{E}[\psi(\theta)]
$$




$$
\begin{aligned}
& \leq \mathbb{E}\left[\mathbb{E}\left[\phi_{\theta}(Z) \mid \theta\right]\right]-\mathbb{E}[\psi(\theta)]+\frac{\mathbb{E}\left[\theta^{2}+(1-\theta)^{2}\right]}{n} \\
& =\mathbb{E}\left[\psi\left(\theta+\sqrt{\frac{\theta(1-\theta)}{n}} Z\right)\right]-\mathbb{E}[\psi(\theta)]+\frac{\mathbb{E}\left[\theta^{2}+(1-\theta)^{2}\right]}{n} \\
& \leq d_{W}\left(\theta, \theta+\sqrt{\frac{\theta(1-\theta)}{n}} Z\right)+\frac{\mathbb{E}\left[\theta^{2}+(1-\theta)^{2}\right]}{n} .
\end{aligned}
$$

Taking the supremum over all $\psi \in \operatorname{Lip}(1)$, we get

$$
d_{W}\left(\bar{X}_{n}, \theta\right) \leq d_{W}\left(\theta+\sqrt{\frac{\theta(1-\theta)}{n}} Z, \theta\right)+\frac{\mathbb{E}\left[\theta^{2}+(1-\theta)^{2}\right]}{n} .
$$

In a similar way, one can show that

$$
d_{W}\left(\theta+\sqrt{\frac{\theta(1-\theta)}{n}} Z, \theta\right) \leq d_{W}\left(\bar{X}_{n}, \theta\right)+\frac{\mathbb{E}\left[\theta^{2}+(1-\theta)^{2}\right]}{n} .
$$

This completes the proof.

The same argument as in the proof of Proposition 3.1 shows that

$$
d_{W}\left(\theta+\sqrt{\frac{\theta(1-\theta)}{n}} Z, \theta\right) \geq \frac{C}{n},
$$

for some $C>0$. This together with Propositions 3.1 and 3.3 leads to the next corollary.

Corollary 3.4. If $\mu(\{0,1\})=0$, and if $Z$ stands for a standard normal random variable independent of $\theta$, then

$$
d_{W}\left(\bar{X}_{n}, \theta\right) \cong d_{W}\left(\theta+\sqrt{\frac{\theta(1-\theta)}{n}} Z, \theta\right) .
$$

We are left with the following question : given $Z$ a standard normal random variable independent of $\theta$, how does the quantity

$$
d_{W}\left(\theta+\sqrt{\frac{\theta(1-\theta)}{n}} Z, \theta\right)
$$

behave as $n$ tends to infinity? To our knowledge, this kind of question has not yet been investigated in the literature. The answer is non-trivial and heavily depends on the law of $\theta$. For instance, when $\theta$ has the Beta distribution, we know from Goldstein and Reinert (2013) (and Corollary 3.4) that $d_{W}\left(\theta+\sqrt{\frac{\theta(1-\theta)}{n}} Z, \theta\right) \cong 1 / n$. As we will see in section 5 , this is not true in general, even if $\theta$ has a density with respect to the Lebesgue measure.

However, in the next section, we show that $d_{W}\left(\bar{X}_{n}, \theta\right) \cong 1 / n$ whenever $\theta$ has a smooth density whose derivative satisfies some integrability property. This includes the case of the Beta distribution. 


\section{Bounds in the case of a smooth density}

4.1. A general bound. The main result of this section is the following Proposition.

Proposition 4.1. Assume the law $\mu$ of $\theta$ has a smooth density $p$ on $(0,1)$ satisfying $\int_{0}^{1} u(1-u)\left|p^{\prime}(u)\right| d u<\infty$. Let $Z$ be a standard normal random variable independent of $\theta$. Then

$$
d_{W}\left(\theta+\sqrt{\frac{\theta(1-\theta)}{n}} Z, \theta\right) \leq \frac{C(\mu)}{n},
$$

where

$$
C(\mu)=\int_{0}^{1}|1-2 u| p(u) d u+\int_{0}^{1} u(1-u)\left|p^{\prime}(u)\right| d u+\frac{3}{\sqrt{2 \pi e}} .
$$

Proof: The proof is rather calculatory and relies on the representation (2.3) of the Wasserstein distance. Let us give some notations first.

- $f(x)=\sqrt{x(1-x)}, x \in(0,1)$.

- $\omega:=x \mapsto \frac{1}{\sqrt{2 \pi}} e^{-x^{2} / 2}$ is the probability density function of a standard normal random variable.

- For a real-valued random variable $X, F_{X}$ denotes its cumulative distribution function.

- $\forall t \geq 0, G(t)=1-F_{Z}(t)=F_{Z}(-t)=\int_{t}^{+\infty} \omega(x) d x$.

We have

$$
d_{W}\left(\theta+\frac{f(\theta)}{\sqrt{n}} Z, \theta\right)=\int_{\mathbb{R}}\left|\mathbb{P}\left[\theta+\frac{f(\theta)}{\sqrt{n}} Z \leq x\right]-\mathbb{P}[\theta \leq x]\right| d x
$$

and

$$
\begin{aligned}
& \mathbb{P}\left[\theta+\sqrt{\frac{\theta(1-\theta)}{n}} Z \leq x\right]-\mathbb{P}[\theta \leq x] \\
& =\int_{0}^{1}\left(\mathbb{P}\left[t+\frac{f(t)}{\sqrt{n}} Z \leq x\right]-\mathbf{1}_{t \leq x}\right) p(t) d t \\
& =\int_{0}^{1}\left(F_{Z}\left(\frac{\sqrt{n}}{f(t)}(x-t)\right)-\mathbf{1}_{t \leq x}\right) p(t) d t .
\end{aligned}
$$

We split the integral (4.2) in several parts, according to the range of $x$.

Case 1 : $0 \leq x \leq 1 / 2$. In this case we write

$$
\begin{aligned}
& \mathbb{P}\left[\theta+\sqrt{\frac{\theta(1-\theta)}{n}} Z \leq x\right]-\mathbb{P}[\theta \leq x] \\
& =\int_{0}^{x}\left(F_{Z}\left(\frac{\sqrt{n}}{f(t)}(x-t)\right)-1\right) p(t) d t+\int_{x}^{1} F_{Z}\left(\frac{\sqrt{n}}{f(t)}(x-t)\right) p(t) d t \\
& =-\int_{0}^{x} G\left(\frac{\sqrt{n}}{f(t)}(x-t)\right) p(t) d t+\int_{x}^{1} G\left(\frac{\sqrt{n}}{f(t)}(t-x)\right) p(t) d t \\
& =-\int_{0}^{x} G\left(\frac{\sqrt{n}}{f(x-t)} t\right) p(x-t) d t+\int_{0}^{1-x} G\left(\frac{\sqrt{n}}{f(t+x)} t\right) p(t+x) d t \\
& =\int_{0}^{x}\left[G\left(\frac{\sqrt{n}}{f(x+t)} t\right) p(x+t)-G\left(\frac{\sqrt{n}}{f(x-t)} t\right) p(x-t)\right] d t
\end{aligned}
$$




$$
\begin{aligned}
& +\int_{x}^{1-x} G\left(\frac{\sqrt{n} t}{f(t+x)}\right) p(t+x) d t \\
= & \frac{1}{\sqrt{n}} \int_{0}^{x \sqrt{n}}\left[G\left(\frac{t}{f\left(x+\frac{t}{\sqrt{n}}\right)}\right) p\left(x+\frac{t}{\sqrt{n}}\right)-G\left(\frac{t}{f\left(x-\frac{t}{\sqrt{n}}\right)}\right) p\left(x-\frac{t}{\sqrt{n}}\right)\right] d t \\
& +\int_{x}^{1-x} G\left(\frac{\sqrt{n} t}{f(x+t)}\right) p(x+t) d t
\end{aligned}
$$

Define, for $(u, t) \in(0,1) \times(0,+\infty)$,

$$
H(u, t)=G\left(\frac{t}{f(u)}\right) p(u) .
$$

The function $H$ has a derivative with respect to its first argument and a direct computation yields

$$
\partial_{1} H(u, t)=t \frac{f^{\prime}(u)}{f^{2}(u)} \omega\left(\frac{t}{f(u)}\right) p(u)+G\left(\frac{t}{f(u)}\right) p^{\prime}(u) .
$$

Thus,

$$
\begin{aligned}
\mathbb{P} & {\left[\theta+\sqrt{\frac{\theta(1-\theta)}{n}} Z \leq x\right]-\mathbb{P}[\theta \leq x] } \\
= & \frac{1}{\sqrt{n}} \int_{0}^{x \sqrt{n}}\left[H\left(x+\frac{t}{\sqrt{n}}, t\right)-H\left(x-\frac{t}{\sqrt{n}}, t\right)\right] d t+\int_{x}^{1-x} G\left(\frac{\sqrt{n} t}{f(x+t)}\right) p(x+t) d t \\
= & \frac{1}{\sqrt{n}} \int_{0}^{x \sqrt{n}} \int_{x-\frac{t}{\sqrt{n}}}^{x+\frac{t}{\sqrt{n}}} \partial_{1} H(u, t) d u d t+\int_{x}^{1-x} G\left(\frac{\sqrt{n} t}{f(x+t)}\right) p(x+t) d t \\
= & \frac{1}{\sqrt{n}}\left[\int_{0}^{x \sqrt{n}} \int_{x-\frac{t}{\sqrt{n}}}^{x+\frac{t}{\sqrt{n}}} t \frac{f^{\prime}(u)}{f^{2}(u)} \omega\left(\frac{t}{f(u)}\right) p(u) d u d t\right. \\
& \left.+\int_{0}^{x \sqrt{n}} \int_{x-\frac{t}{\sqrt{n}}}^{x+\frac{t}{\sqrt{n}}} G\left(\frac{t}{f(u)}\right) p^{\prime}(u) d u d t\right] \\
& +\int_{x}^{1-x} G\left(\frac{\sqrt{n} t}{f(x+t)}\right) p(x+t) d t \\
:= & A_{1}(x)+A_{2}(x)+A_{3}(x)
\end{aligned}
$$

We will bound separately the integrals of the absolute values of $A_{1}, A_{2}$ and $A_{3}$ on $(0,1 / 2)$.

First we focus on $A_{1}$.

$$
\begin{aligned}
\int_{0}^{1 / 2}\left|A_{1}(x)\right| d x & =\frac{1}{\sqrt{n}} \int_{0}^{1 / 2}\left|\int_{0}^{x \sqrt{n}} \int_{x-\frac{t}{\sqrt{n}}}^{x+\frac{t}{\sqrt{n}}} t \frac{f^{\prime}(u)}{f^{2}(u)} \omega\left(\frac{t}{f(u)}\right) p(u) d u d t\right| d x \\
& \leq \frac{1}{\sqrt{n}} \int_{0}^{1 / 2} \int_{0}^{x \sqrt{n}} \int_{x-\frac{t}{\sqrt{n}}}^{x+\frac{t}{\sqrt{n}}} t \frac{\left|f^{\prime}(u)\right|}{f^{2}(u)} \omega\left(\frac{t}{f(u)}\right) p(u) d u d t d x
\end{aligned}
$$

We apply Fubini's theorem with a (possibly) larger region of integration, using the fact that

$$
\left\{(x, t, u) \mid 0 \leq x \leq \frac{1}{2}, 0 \leq t \leq x \sqrt{n}, x-\frac{t}{\sqrt{n}} \leq u \leq x+\frac{t}{\sqrt{n}}\right\}
$$




$$
\subset\left\{(x, t, u) \mid 0 \leq u \leq 1,0 \leq t \leq \frac{\sqrt{n}}{2}, u-\frac{t}{\sqrt{n}} \leq x \leq u+\frac{t}{\sqrt{n}}\right\} .
$$

This yields

$$
\begin{aligned}
\int_{0}^{1 / 2}\left|A_{1}(x)\right| d x & \leq \frac{1}{\sqrt{n}} \int_{0}^{1} p(u) \frac{\left|f^{\prime}(u)\right|}{f^{2}(u)} \int_{0}^{\sqrt{n} / 2} \int_{u-\frac{t}{\sqrt{n}}}^{u+\frac{t}{\sqrt{n}}} d x t \omega\left(\frac{t}{f(u)}\right) d u d t \\
& =\frac{2}{n} \int_{0}^{1} p(u) \frac{\left|f^{\prime}(u)\right|}{f^{2}(u)} \int_{0}^{\sqrt{n} / 2} t^{2} \omega\left(\frac{t}{f(u)}\right) d t d u \\
& =\frac{2}{n} \int_{0}^{1} p(u) \frac{\left|f^{\prime}(u)\right|}{f^{2}(u)} f^{3}(u) \int_{0}^{\frac{\sqrt{n}}{2 f(u)}} t^{2} \omega(t) d t d u \\
& \leq \frac{1}{n} \int_{0}^{1} p(u)\left|\left(f^{2}(u)\right)^{\prime}\right| \int_{0}^{+\infty} t^{2} \omega(t) d t d u \\
& =\frac{1}{2 n} \int_{0}^{1} p(u)|1-2 u| d u
\end{aligned}
$$

A similar computation yields

$$
\begin{aligned}
\int_{0}^{1 / 2}\left|A_{2}(x)\right| d x & =\frac{1}{\sqrt{n}} \int_{0}^{1 / 2}\left|\int_{0}^{x \sqrt{n}} \int_{x-\frac{t}{\sqrt{n}}}^{x+\frac{t}{\sqrt{n}}} G\left(\frac{t}{f(u)}\right) p^{\prime}(u) d u d t\right| d x \\
& \leq \frac{1}{\sqrt{n}} \int_{0}^{1}\left|p^{\prime}(u)\right| \int_{0}^{\sqrt{n} / 2} \int_{u-\frac{t}{\sqrt{n}}}^{u+\frac{t}{\sqrt{n}}} d x G\left(\frac{t}{f(u)}\right) d t d u \\
& =\frac{2}{n} \int_{0}^{1}\left|p^{\prime}(u)\right| \int_{0}^{\sqrt{n} / 2} t G\left(\frac{t}{f(u)}\right) d t d u \\
& =\frac{2}{n} \int_{0}^{1}\left|p^{\prime}(u)\right| f^{2}(u) \int_{0}^{\frac{\sqrt{n}}{2 f(u)}} t G(t) d t d u \\
& \leq \frac{2}{n} \int_{0}^{1}\left|p^{\prime}(u)\right| u(1-u) \int_{0}^{+\infty} t G(t) d t d u \\
& =\frac{1}{2 n} \int_{0}^{1}\left|p^{\prime}(u)\right| u(1-u) d u,
\end{aligned}
$$

where we used the fact that $\int_{0}^{+\infty} t G(t) d t=1 / 4$ (for instance from an integration by parts).

As for $A_{3}$, using Fubini's theorem again we have

$$
\begin{aligned}
\int_{0}^{1 / 2}\left|A_{3}(x)\right| d x & =\int_{0}^{1 / 2} \int_{x}^{1-x} G\left(\frac{\sqrt{n}}{f(t+x)} t\right) p(t+x) d t d x \\
& =\int_{0}^{1 / 2} \int_{2 x}^{1} G\left(\frac{\sqrt{n}}{f(t)}(t-x)\right) p(t) d t d x \\
& =\int_{0}^{1} p(t) \int_{0}^{t / 2} G\left(\frac{\sqrt{n}}{f(t)}(t-x)\right) d x d t \\
& =\int_{0}^{1} p(t) \int_{t / 2}^{t} G\left(\frac{\sqrt{n}}{f(t)} x\right) d x d t
\end{aligned}
$$




$$
\begin{aligned}
& =\frac{1}{\sqrt{n}} \int_{0}^{1} p(t) f(t) \int_{\frac{\sqrt{n} t}{2 f(t)}}^{\frac{\sqrt{n} t}{f(t)}} G(x) d x d t \\
& \leq \frac{1}{\sqrt{n}} \int_{0}^{1} p(t) f(t) \int_{\frac{\sqrt{n} t}{2 f(t)}}^{+\infty} G(x) d x d t
\end{aligned}
$$

Now, integrating by parts we have $\int_{y}^{+\infty} G(u) d u=\omega(y)-y G(y) \leq \omega(y)$, so that

$$
\forall y>0, \quad \int_{y}^{+\infty} G(u) d u \leq \omega(y) \leq \frac{1}{y \sqrt{2 \pi e}},
$$

an inequality easily shown for instance by studying the function $y \mapsto y \omega(y)$. This yields

$$
\int_{0}^{1 / 2}\left|A_{3}(x)\right| d x \leq \frac{2}{n \sqrt{2 \pi e}} \int_{0}^{1} p(t) \frac{f^{2}(t)}{t} d t=\frac{\sqrt{2}}{n \sqrt{\pi e}} \int_{0}^{1} p(t)(1-t) d t
$$

To sum up,

$$
\begin{aligned}
& \int_{0}^{1 / 2}\left|\mathbb{P}\left[\theta+\sqrt{\frac{\theta(1-\theta)}{n}} Z \leq x\right]-\mathbb{P}[\theta \leq x]\right| d x \\
& \leq \frac{1}{2 n}\left[\int_{0}^{1} p(u)|1-2 u| d u+\int_{0}^{1} u(1-u)\left|p^{\prime}(u)\right| d u+\frac{2 \sqrt{2}}{\sqrt{\pi e}} \int_{0}^{1} p(u)(1-u) d u\right] .
\end{aligned}
$$

Case 2: $x \leq 0$. In this case, from (4.3) we have

$$
\mathbb{P}\left[\theta+\sqrt{\frac{\theta(1-\theta)}{n}} Z \leq x\right]-\mathbb{P}[\theta \leq x]=\int_{0}^{1} G\left(\frac{\sqrt{n}}{f(t)}(t-x)\right) p(t) d t,
$$

so that

$$
\begin{aligned}
& \int_{-\infty}^{0}\left|\mathbb{P}\left[\theta+\sqrt{\frac{\theta(1-\theta)}{n}} Z \leq x\right]-\mathbb{P}[\theta \leq x]\right| d x \\
& =\int_{-\infty}^{0} \int_{0}^{1} G\left(\frac{\sqrt{n}}{f(t)}(t-x)\right) p(t) d t d x \\
& =\int_{0}^{+\infty} \int_{0}^{1} G\left(\frac{\sqrt{n}}{f(t)}(t+x)\right) p(t) d t d x \\
& =\int_{0}^{1} p(t) \int_{0}^{+\infty} G\left(\frac{\sqrt{n}}{f(t)}(t+x)\right) d x d t \\
& =\frac{1}{\sqrt{n}} \int_{0}^{1} p(t) f(t) \int_{\frac{\sqrt{n} t}{f(t)}}^{+\infty} G(u) d u d t .
\end{aligned}
$$

Using (4.4), we obtain

$$
\begin{aligned}
& \int_{-\infty}^{0}\left|\mathbb{P}\left[\theta+\sqrt{\frac{\theta(1-\theta)}{n}} Z \leq x\right]-\mathbb{P}[\theta \leq x]\right| d x \\
& \leq \frac{1}{n \sqrt{2 \pi e}} \int_{0}^{1} p(t) \frac{f^{2}(t)}{t} d t=\frac{1}{n \sqrt{2 \pi e}} \int_{0}^{1} p(t)(1-t) d t .
\end{aligned}
$$


From (4.5) and (4.6) we get

$$
\begin{aligned}
& \int_{-\infty}^{1 / 2}\left|\mathbb{P}\left[\theta+\sqrt{\frac{\theta(1-\theta)}{n}} Z \leq x\right]-\mathbb{P}[\theta \leq x]\right| d x \\
& \leq \frac{1}{2 n}\left[\int_{0}^{1} p(u)|1-2 u| d u+\int_{0}^{1} u(1-u)\left|p^{\prime}(u)\right| d u+\frac{3 \sqrt{2}}{\sqrt{\pi e}} \int_{0}^{1} p(u)(1-u) d u\right] .
\end{aligned}
$$

Case 3: $x \geq 1 / 2$. In this case, we can use the symmetry of $f$ and $Z$ and the bound found for $x \leq 1 / 2$. More precisely, let $\theta_{1}=1-\theta$. Then, since $f(1-t)=f(t)$,

$$
\begin{aligned}
& \mathbb{P}\left[\theta+\frac{f(\theta)}{\sqrt{n}} Z \leq x\right]-\mathbb{P}[\theta \leq x] \\
& =\mathbb{P}\left[1-\theta-\frac{f(1-\theta)}{\sqrt{n}} Z \geq 1-x\right]-\mathbb{P}[1-\theta \geq 1-x] \\
& =\mathbb{P}\left[\theta_{1}-\frac{f\left(\theta_{1}\right)}{\sqrt{n}} Z \geq 1-x\right]-\mathbb{P}\left[\theta_{1} \geq 1-x\right] \\
& =\mathbb{P}\left[\theta_{1}+\frac{f\left(\theta_{1}\right)}{\sqrt{n}} Z \geq 1-x\right]-\mathbb{P}\left[\theta_{1} \geq 1-x\right] \\
& =\mathbb{P}\left[\theta_{1} \leq 1-x\right]-\mathbb{P}\left[\theta_{1}+\frac{f\left(\theta_{1}\right)}{\sqrt{n}} Z \leq 1-x\right] .
\end{aligned}
$$

Thus,

$$
\begin{aligned}
& \int_{1 / 2}^{+\infty}\left|\mathbb{P}\left[\theta+\frac{f(\theta)}{\sqrt{n}} Z \leq x\right]-\mathbb{P}[\theta \leq x]\right| d x \\
& =\int_{1 / 2}^{+\infty}\left|\mathbb{P}\left[\theta_{1}+\frac{f\left(\theta_{1}\right)}{\sqrt{n}} Z \leq 1-x\right]-\mathbb{P}\left[\theta_{1} \leq 1-x\right]\right| d x \\
& =\int_{-\infty}^{1 / 2}\left|\mathbb{P}\left[\theta_{1}+\frac{f\left(\theta_{1}\right)}{\sqrt{n}} Z \leq x\right]-\mathbb{P}\left[\theta_{1} \leq x\right]\right| d x .
\end{aligned}
$$

Now we can use the bound in (4.7) with the transformation $p(u) \rightarrow p(1-u)$, to obtain (after a change of variables $v=1-u$ in the integrals) :

$$
\begin{aligned}
& \int_{1 / 2}^{+\infty}\left|\mathbb{P}\left[\theta+\sqrt{\frac{\theta(1-\theta)}{n}} Z \leq x\right]-\mathbb{P}[\theta \leq x]\right| d x \\
& \leq \frac{1}{2 n}\left[\int_{0}^{1} p(u)|1-2 u| d u+\int_{0}^{1} u(1-u)\left|p^{\prime}(u)\right| d u+\frac{3 \sqrt{2}}{\sqrt{\pi e}} \int_{0}^{1} p(u) u d u\right] .
\end{aligned}
$$

The proof follows from the last inequality and (4.7).

The proof of our main theorem follows easily.

Proof of Theorem 1.2: The first inequality in (1.5) is just a restatement of Proposition 3.1, whereas the upper bound follows from Propositions 3.3 and 4.1. 
4.2. Application to the Beta distribution. We specialize the result of Theorem 1.2 to the case of the Beta distribution. We explicit the bounds in (1.5) when the density $p$ is given by (4.8). As a by-product, we obtain bounds of the optimal order with explicit constants for the distance of the scaled number of white balls drawn from a Pólya-Eggenberger urn to its limiting distribution. As said before, such bounds were already obtained in Goldstein and Reinert (2013), with explicit constants as well. This will allow us to numerically compare the constants found in this article and the ones in Goldstein and Reinert (2013).

Let us first describle the model. At time 0 , an urn contains $A \geq 1$ white balls and $B \geq 1$ black balls and at every positive integer time, a ball is randomly drawn from the urn (independently of the past) and replaced along with $m \geq 1$ additional balls of the same color. Then, defining $X_{n}=1$ if a white ball is drawn at time $n$ and $X_{n}=0$ otherwise, it is well known that (1.1) holds with $\mu$ being the Beta distribution with parameters $A / m$ and $B / m$. Here, the Beta distribution with parameters $\alpha>0$ and $\beta>0$ is the probability measure with density

$$
p_{\alpha, \beta}(x)=\frac{1}{B(\alpha, \beta)} x^{\alpha-1}(1-x)^{\beta-1} \mathbf{1}_{(0,1)}(x),
$$

where $B(\alpha, \beta)=\int_{0}^{1} t^{\alpha-1}(1-t)^{\beta-1} d t$ is the Beta function and $\mathbf{1}_{E}$ the indicator function of the set $E$.

We begin with a Lemma which can be shown by elementary computations. Recall $B$ denotes the Beta function and we denote by $B_{i}$ the incomplete Beta function : for $x \in[0,1], \alpha>0, \beta>0, B_{i}(x, \alpha, \beta)=\int_{0}^{x} t^{\alpha-1}(1-t)^{\beta-1} d t$.

Lemma 4.2. Let $\theta$ have the Beta distribution with parameters $\alpha$ and $\beta$. For $a, b \in \mathbb{R}$ let

$$
F(\alpha, \beta, a, b)=\mathbb{E}[|a \theta+b|]
$$

Then, if $a>0$,

$$
\begin{aligned}
& F(\alpha, \beta, a, b) \\
& = \begin{cases}\frac{1}{B(\alpha, \beta)}\left[-2 a B_{i}(-b / a, \alpha+1, \beta)\right. & \text { if }-a<b \leq 0, \\
\frac{\left.-2 b B_{i}(-b / a, \alpha, \beta)+a B(\alpha+1, \beta)+b B(\alpha, \beta)\right]}{\frac{1}{B(\alpha, \beta)}[a B(\alpha+1, \beta)+b B(\alpha, \beta)]} & \text { if } b>0, \\
-\frac{1}{B(\alpha, \beta)}[a B(\alpha+1, \beta)+b B(\alpha, \beta)] & \text { if } b \leq-a .\end{cases}
\end{aligned}
$$

If $a=0, F(\alpha, \beta, 0, b)=|b|$, and if $a<0, F(\alpha, \beta, a, b)=F(\alpha, \beta,-a,-b)$.

Proof: If $-a<b \leq 0$, then $0 \leq-\frac{b}{a}<1$, so that

$$
\begin{aligned}
& \mathbb{E}[|a \theta+b|]= \\
= & \frac{1}{B(\alpha, \beta)}\left[\int_{0}^{-b / a}(-a t-b) t^{\alpha-1}(1-t)^{\beta-1} d t+\int_{-b / a}^{1}(a t+b) t^{\alpha-1}(1-t)^{\beta-1} d t\right] .
\end{aligned}
$$

An expansion and straightforward calculations give the result in this case. The other cases are dealt with similarly.

Proposition 4.3. Assume that the law $\mu$ of $\theta$ is the Beta distribution with parameters $\alpha$ and $\beta$. Then

$$
d_{W}\left(\bar{X}_{n}, \theta\right) \leq \frac{C_{\alpha, \beta}}{n}
$$


where

$C_{\alpha, \beta}=\frac{B(\alpha+2, \beta)+B(\alpha, \beta+2)}{B(\alpha, \beta)}+F(\alpha, \beta, 2,-1)+F(\alpha, \beta, \alpha+\beta-2,1-\alpha)+\frac{3}{\sqrt{2 \pi e}}$,

and $F$ is defined in Lemma 4.2.

Proof: If $p$ is the density of the Beta distribution defined in (4.8), it is clear that $p$ satisfies the assumptions of Theorem 1.2. Note that $\int_{0}^{1}|1-2 u| p(u) d u=$ $\mathbb{E}[|1-2 \theta|]=F(\alpha, \beta, 2,-1)$. It is straightforward to show that $\int_{0}^{1}\left(u^{2}+(1-\right.$ $\left.u)^{2}\right) p(u) d u=\frac{B(\alpha+2, \beta)+B(\alpha, \beta+2)}{B(\alpha, \beta)}$. Moreover,

$$
\begin{aligned}
\int_{0}^{1} u(1-u)\left|p^{\prime}(u)\right| d u & =\int_{0}^{1} u(1-u)\left|\frac{\alpha-1}{u}-\frac{\beta-1}{1-u}\right| p(u) d u \\
& =\int_{0}^{1}|(\alpha-1)(1-u)-(\beta-1) u| p(u) d u \\
& =\int_{0}^{1}|(\alpha+\beta-2) u+1-\alpha| p(u) d u \\
& =F(\alpha, \beta, \alpha+\beta-2,1-\alpha),
\end{aligned}
$$

proving our claim.

Corollary 4.4. In a Pólya-Eggenberg urn containing initially $A$ white balls and $B$ black balls, and where at each draw a ball is replaced along with $m$ balls of the same color, let $\bar{X}_{n}$ be the scaled number of white balls in $n$ draws. Let $\theta$ have the Beta distribution with parameters $A / m$ and $B / m$. Then

$$
d_{W}\left(\bar{X}_{n}, \theta\right) \leq \frac{C_{A / m, B / m}}{n},
$$

where $C_{\alpha, \beta}$ is defined in (4.10).

Now, let us compare this result with the one of Goldstein and Reinert. We plot the ratio of the constant $C_{A / m, B / m}$ to the one obtained in Goldstein and Reinert (2013), Theorem 1.1, for values of $A / m$ and $B / m$ ranging from $10^{-5}$ to 3 .

As we can see, our constant $C_{A / m, B / m}$ is at least half that of Goldstein and Reinert (2013) for the set of parameters we chose; the ratio seems to go to zero as $A / m$ or $B / m$ become large.

\section{The rate $1 / n^{\delta}$ is possible for any $1 / 2 \leq \delta \leq 1$}

We saw in Proposition 4.1 that a sufficient condition to get a rate of convergence of the order $1 / n$ for $d_{W}\left(\theta+\sqrt{\frac{\theta(1-\theta)}{n}} Z, \theta\right)$ is that $\mu$ is absolutely continuous with density on $\quad p \quad(0,1) \quad$ satisfying $\int_{0}^{1} u(1-u)\left|p^{\prime}(u)\right| d u<\infty$. The goal of this section is to show that this is not true anymore with the weaker asumption that $\mu$ is simply absolutely continuous. Actually, for each $\delta \in(1 / 2,1)$, we give an example of a measure $\mu$ with a density on $(0,1)$ such that $d_{W}\left(\theta+\sqrt{\frac{\theta(1-\theta)}{n}} Z, \theta\right)$ is of the order $1 / n^{\delta}$. This is the content of the two following propositions. 


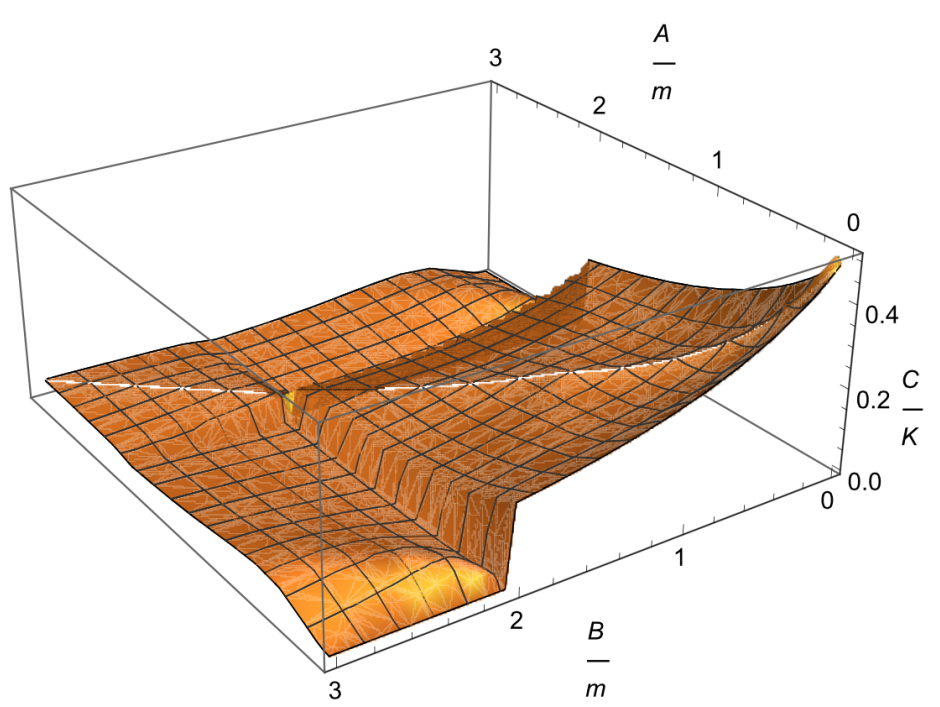

Figure 4.1. $C_{A / m, B / m} / K_{A / m, B / m}$ where $K_{A / m, B / m}$ is defined in Goldstein and Reinert (2013), Theorem 1.1. $(A / m, B / m) \in$ $\left[10^{-5}, 3\right]^{2}$.

Proposition 5.1. Let $\gamma \in(0,1)$ and suppose that $\theta$ has the density $p(x)=C_{p}(x-$ $\left.\frac{1}{2}\right)^{\gamma-1} \mathbf{1}_{(1 / 2,3 / 4)}(x)\left(C_{p}\right.$ is a normalizing constant). Let $\left(X_{k}\right)_{k \geq 1}$ be a Bernoulli sequence with prior $\theta$. Then

$$
d_{W}\left(\bar{X}_{n}, \theta\right)=\mathcal{O}\left(\frac{1}{n^{\frac{1+\gamma}{2}}}\right) .
$$

Proof: From Proposition 3.3, it is sufficient to show that

$$
d_{W}\left(\theta+\sqrt{\frac{\theta(1-\theta)}{n}} Z, \theta\right)=\mathcal{O}\left(\frac{1}{n^{\frac{1+\gamma}{2}}}\right),
$$

where $Z$ stands for a normal random variable independent of $\theta$. The proof is similar to the one of Proposition 4.1; we use here the same notations and do not give all the details in the calculations. In the following, $M_{1}$ and $M_{2}$ are generic positive constants that may vary from line to line. We have

$$
d_{W}\left(\theta+\frac{f(\theta)}{\sqrt{n}} Z, \theta\right)=\int_{\mathbb{R}}\left|\mathbb{P}\left[\theta+\frac{f(\theta)}{\sqrt{n}} Z \leq x\right]-\mathbb{P}[\theta \leq x]\right| d x .
$$

Define $\tilde{\theta}=4\left(\theta-\frac{1}{2}\right)$, so that $\tilde{\theta}$ has density equal to $\tilde{C}_{p} x^{\gamma-1} \mathbf{1}_{(0,1)}(x)\left(\tilde{C}_{p}\right.$ normalizing constant). For $x \in(0,1)$, let $\tilde{f}(x)=4 f\left(1 / 2+\frac{x}{4}\right)$. We have

$$
\int_{\mathbb{R}}\left|\mathbb{P}\left[\theta+\frac{f(\theta)}{\sqrt{n}} Z \leq x\right]-\mathbb{P}[\theta \leq x]\right| d x
$$




$$
\begin{aligned}
& =\int_{\mathbb{R}}\left|\mathbb{P}\left[\tilde{\theta}+\frac{\tilde{f}(\tilde{\theta})}{\sqrt{n}} Z \leq 4(x-1 / 2)\right]-\mathbb{P}[\tilde{\theta} \leq 4(x-1 / 2)]\right| d x \\
& =\frac{1}{4} \int_{\mathbb{R}}\left|\mathbb{P}\left[\tilde{\theta}+\frac{\tilde{f}(\tilde{\theta})}{\sqrt{n}} Z \leq x\right]-\mathbb{P}[\tilde{\theta} \leq x]\right| d x
\end{aligned}
$$

Thus it suffices to show that the last quantity is a $\mathcal{O}\left(\frac{1}{n^{\frac{1+\gamma}{2}}}\right)$.

Case 1: $0 \leq x \leq 1 / 2$. In this case

$$
\begin{aligned}
& \frac{1}{\tilde{C}_{p}}\left(\mathbb{P}\left[\tilde{\theta}+\frac{\tilde{f}(\tilde{\theta})}{\sqrt{n}} Z \leq x\right]-\mathbb{P}[\tilde{\theta} \leq x]\right) \\
& =\int_{0}^{x}\left(G\left(\frac{\sqrt{n} t}{\tilde{f}(x+t)}\right)(x+t)^{\gamma-1}-G\left(\frac{\sqrt{n} t}{\tilde{f}(x-t)}\right)(x-t)^{\gamma-1}\right) d t \\
& +\int_{x}^{1-x} G\left(\frac{\sqrt{n} t}{\tilde{f}(x+t)}\right)(x+t)^{\gamma-1} d t .
\end{aligned}
$$

If $\tilde{H}(u, t)=G\left(\frac{t}{\tilde{f}(u)}\right) u^{\gamma-1}$, then

$$
\partial_{1} \tilde{H}(u, t)=t \frac{\tilde{f}^{\prime}(u)}{\tilde{f}^{2}(u)} \omega\left(\frac{t}{\tilde{f}(u)}\right) u^{\gamma-1}-(1-\gamma) G\left(\frac{t}{\tilde{f}(u)}\right) u^{\gamma-2} .
$$

It is clear from the definition of $f$ and $\tilde{f}$ that $0<m_{1} \leq \tilde{f}(u) \leq 1$ and $\left|\tilde{f}^{\prime}(u)\right| \leq m_{2}$ for some constants $m_{1}$ and $m_{2}$. Thus

$$
\left|\partial_{1} \tilde{H}(u, t)\right| \leq M_{1} t \omega(t) u^{\gamma-1}+M_{2} G(t) u^{\gamma-2} .
$$

We get

$$
\begin{aligned}
& \int_{0}^{1 / 2} \int_{0}^{x}\left|G\left(\frac{\sqrt{n} t}{\tilde{f}(x+t)}\right)(x+t)^{\gamma-1}-G\left(\frac{\sqrt{n} t}{\tilde{f}(x-t)}\right)(x-t)^{\gamma-1}\right| d t d x \\
& \leq \int_{0}^{1 / 2} \int_{0}^{x} \int_{x-t}^{x+t}\left(M_{1} \sqrt{n} t \omega(\sqrt{n} t) u^{\gamma-1}+M_{2} G(\sqrt{n} t) u^{\gamma-2}\right) d u d t d x
\end{aligned}
$$

However,

$$
\begin{aligned}
& \int_{0}^{1 / 2} \int_{0}^{x} \int_{x-t}^{x+t} \sqrt{n} t \omega(\sqrt{n} t) u^{\gamma-1} d u d t d x \\
& =\frac{1}{n^{1+\gamma / 2}} \int_{0}^{\sqrt{n} / 2} \int_{0}^{x} \int_{x-t}^{x+t} t \omega(t) u^{\gamma-1} d u d t d x \\
& =\frac{1}{n^{1+\gamma / 2}} \int_{0}^{\sqrt{n} / 2} t \omega(t) \int_{t}^{\sqrt{n} / 2} \int_{x-t}^{x+t} u^{\gamma-1} d u d x d t \\
& \leq \frac{1}{n^{1+\gamma / 2}} \int_{0}^{\sqrt{n} / 2} t \omega(t) \int_{0}^{t+\sqrt{n} / 2} u^{\gamma-1} \int_{u-t}^{u+t} d x d u d t \\
& \leq \frac{2}{n^{1+\gamma / 2}} \int_{0}^{\sqrt{n} / 2} t^{2} \omega(t) \int_{0}^{\sqrt{n}} u^{\gamma-1} d u d t \\
& \leq \frac{M_{1}}{n} \int_{0}^{+\infty} t^{2} \omega(t) d t .
\end{aligned}
$$


On the other hand,

$$
\begin{aligned}
& \int_{0}^{1 / 2} \int_{0}^{x} \int_{x-t}^{x+t} G(\sqrt{n} t) u^{\gamma-2} d u d t d x \\
& =\frac{1}{n^{\frac{1+\gamma}{2}}} \int_{0}^{\sqrt{n} / 2} \int_{0}^{x} G(t) \int_{x-t}^{x+t} u^{\gamma-2} d u d t d x \\
& \leq \frac{1}{n^{\frac{1+\gamma}{2}}} \int_{0}^{+\infty} \int_{0}^{x} G(t) \int_{x-t}^{x+t} u^{\gamma-2} d u d t d x \\
& =\frac{1}{n^{\frac{1+\gamma}{2}}} \int_{0}^{+\infty} G(t) \int_{t}^{+\infty} \int_{-t}^{t}(u+x)^{\gamma-2} d u d x d t \\
& =\frac{1}{n^{\frac{1+\gamma}{2}}} \int_{0}^{+\infty} G(t) \int_{-t}^{t} \int_{t}^{+\infty}(u+x)^{\gamma-2} d x d u d t \\
& \leq \frac{M_{1}}{n^{\frac{1+\gamma}{2}}} \int_{0}^{+\infty} G(t) \int_{-t}^{t}(t+u)^{\gamma-1} d u d t \\
& \leq \frac{M_{1}}{n^{\frac{1+\gamma}{2}}} \int_{0}^{+\infty} G(t) t^{\gamma} d t .
\end{aligned}
$$

It remains to show that $\int_{0}^{1 / 2} \int_{x}^{1-x} G\left(\frac{\sqrt{n} t}{\tilde{f}(x+t)}\right)(x+t)^{\gamma-1} d t=\mathcal{O}\left(\frac{1}{n^{\frac{1+\gamma}{2}}}\right)$.

$$
\begin{aligned}
& \int_{0}^{1 / 2} \int_{x}^{1-x} G\left(\frac{\sqrt{n} t}{\tilde{f}(x+t)}\right)(x+t)^{\gamma-1} d t d x \\
& \leq \int_{0}^{1 / 2} \int_{2 x}^{1} G(\sqrt{n}(t-x) / 4) t^{\gamma-1} d t d x \\
& =\int_{0}^{1} t^{\gamma-1} \int_{0}^{t / 2} G(\sqrt{n}(t-x) / 4) d x d t \\
& =\int_{0}^{1} t^{\gamma-1} \int_{t / 2}^{t} G(\sqrt{n} x / 4) d x d t \\
& =\frac{1}{n^{\frac{1+\gamma}{2}}} \int_{0}^{\sqrt{n}} t^{\gamma-1} \int_{t / 2}^{t} G(x / 4) d x d t \\
& \leq \frac{1}{n^{\frac{1+\gamma}{2}}} \int_{0}^{+\infty} t^{\gamma-1} \int_{t / 2}^{t} G(x / 4) d x d t
\end{aligned}
$$

so the case $0 \leq x \leq 1 / 2$ is complete.

Case 2: $x \leq 0$. In this case, similarly as Case 2 of the proof of Proposition 4.1, we show that

$$
\begin{aligned}
& \int_{-\infty}^{0}\left|\mathbb{P}\left[\tilde{\theta}+\sqrt{\frac{\tilde{\theta}(1-\tilde{\theta})}{n}} Z \leq x\right]-\mathbb{P}[\tilde{\theta} \leq x]\right| d x \\
& =\int_{0}^{1} t^{\gamma-1} \int_{0}^{+\infty} G\left(\frac{\sqrt{n}}{\tilde{f}(t)}(t+x)\right) d x d t \\
& \leq \int_{0}^{1} t^{\gamma-1} \int_{0}^{+\infty} G(\sqrt{n}(t+x) / 4) d x d t
\end{aligned}
$$




$$
\begin{aligned}
& =\frac{1}{n^{\frac{1+\gamma}{2}}} \int_{0}^{\sqrt{n}} t^{\gamma-1} \int_{t}^{+\infty} G(x / 4) d x d t \\
& \leq \frac{1}{n^{\frac{1+\gamma}{2}}} \int_{0}^{+\infty} t^{\gamma-1} \int_{t}^{+\infty} G(x / 4) d x d t .
\end{aligned}
$$

The bound in the cases $1 / 2 \leq x \leq 1$ and $x \geq 1$ are proved in a similar manner.

Now let us prove that $d_{W}\left(\bar{X}_{n}, \theta\right)$ is also bounded below by a term of the order $\frac{1}{n^{\frac{1+\gamma}{2}}}$.

Proposition 5.2. Under the hypothesis of Proposition 5.1, there exists $C>0$ such that

$$
d_{W}\left(\bar{X}_{n}, \theta\right) \geq \frac{C}{n^{\frac{1+\gamma}{2}}} .
$$

Proof: From Proposition 3.3, it suffices to prove that the same type of bound holds for $d_{W}\left(\theta+\frac{f(\theta)}{\sqrt{n}} Z, \theta\right), Z$ being a standard normal variable independent of $\theta$. We use the dual version of the Wasserstein distance. Let $\psi$ be the 1-Lipschitz function defined by

$$
\psi(x)=\left|x-\frac{1}{2}\right| .
$$

As before, let $f(x)=\sqrt{x(1-x)}$. Then

$$
\begin{aligned}
& \mathbb{E}\left[\psi\left(\theta+\frac{f(\theta)}{\sqrt{n}} Z\right)\right] \\
& =C_{p} \int_{1 / 2}^{3 / 4} \mathbb{E}\left[\left|t+\frac{f(t)}{\sqrt{n}} Z-\frac{1}{2}\right|\right]\left(t-\frac{1}{2}\right)^{\gamma-1} d t \\
& =C_{p} \int_{0}^{1 / 4} t^{\gamma-1} \mathbb{E}\left[\left|t+\frac{f(t+1 / 2)}{\sqrt{n}} Z\right|\right] d t \\
& =C_{p} \int_{0}^{1 / 4} t^{\gamma-1} \frac{f(t+1 / 2)}{\sqrt{n}} \mathbb{E}\left[\left|\frac{\sqrt{n} t}{f(t+1 / 2)}+Z\right|\right] d t .
\end{aligned}
$$

However, a straightforward computation shows that for every $a \in \mathbb{R}$,

$$
\mathbb{E}[|a+Z|]=a\left(1-2 F_{Z}(-a)\right)+2 \omega(a) .
$$

Thus

$$
\begin{aligned}
& \mathbb{E}\left[\psi\left(\theta+\frac{f(\theta)}{\sqrt{n}} Z\right)\right] \\
= & C_{p} \int_{0}^{1 / 4} t^{\gamma-1}\left[t\left(1-2 F_{Z}\left(-\frac{\sqrt{n} t}{f(t+1 / 2)}\right)\right)+\frac{2 f(t+1 / 2)}{\sqrt{n}} \omega\left(\frac{\sqrt{n} t}{f(t+1 / 2)}\right)\right] d t .
\end{aligned}
$$

On the other hand, we have

$$
\mathbb{E}[\psi(\theta)]=C_{p} \int_{0}^{1 / 4} t^{\gamma} d t
$$

We obtain

$$
\mathbb{E}\left[\psi\left(\theta+\frac{f(\theta)}{\sqrt{n}} Z\right)\right]-\mathbb{E}[\psi(\theta)]
$$




$$
\begin{aligned}
& =2 C_{p} \int_{0}^{1 / 4} t^{\gamma-1}\left[-t F_{Z}\left(-\frac{\sqrt{n} t}{f(t+1 / 2)}\right)+\frac{f(t+1 / 2)}{\sqrt{n}} \omega\left(\frac{\sqrt{n} t}{f(t+1 / 2)}\right)\right] d t \\
& =\frac{2 C_{p}}{n^{\frac{\gamma}{2}}} \int_{0}^{\frac{1}{4} \sqrt{n}} t^{\gamma-1}\left[-\frac{t}{\sqrt{n}} F_{Z}\left(-\frac{t}{f\left(\frac{t}{\sqrt{n}}+1 / 2\right)}\right)+\frac{f\left(\frac{t}{\sqrt{n}}+1 / 2\right)}{\sqrt{n}} \omega\left(\frac{t}{f\left(\frac{t}{\sqrt{n}}+1 / 2\right)}\right)\right] d t \\
& =\frac{2 C_{p}}{n^{\frac{1+\gamma}{2}}} \int_{0}^{\frac{1}{4} \sqrt{n}} t^{\gamma-1}\left[-t F_{Z}\left(-\frac{t}{f\left(\frac{t}{\sqrt{n}}+1 / 2\right)}\right)+f\left(\frac{t}{\sqrt{n}}+1 / 2\right) \omega\left(\frac{t}{f\left(\frac{t}{\sqrt{n}}+1 / 2\right)}\right)\right] d t .
\end{aligned}
$$

Now, since $0 \leq f(x) \leq 1$ for every $x \in[0,1]$, we have that

$$
\begin{aligned}
& \left|t^{\gamma-1}\left[-t F_{Z}\left(-\frac{t}{f\left(\frac{t}{\sqrt{n}}+1 / 2\right)}\right)+f\left(\frac{t}{\sqrt{n}}+1 / 2\right) \omega\left(\frac{t}{f\left(\frac{t}{\sqrt{n}}+1 / 2\right)}\right)\right]\right| \\
\leq & t^{\gamma-1}\left[t F_{Z}(-t)+\omega(t)\right],
\end{aligned}
$$

so that the above integral tends to $\int_{0}^{+\infty} t^{\gamma-1}\left[-t F_{Z}(-2 t)+\frac{1}{2} \omega(2 t)\right] d t$ by dominated convergence. It remains to show that this limit is not zero to achieve the proof. Integrating by parts twice, we have

$$
\int_{0}^{+\infty} t^{\gamma} F_{Z}(-2 t) d t=\frac{\gamma}{2(\gamma+1)} \int_{0}^{+\infty} t^{\gamma-1} \omega(2 t) d t
$$

so that

$$
\int_{0}^{+\infty} t^{\gamma-1}\left[-t F_{Z}(-2 t)+\frac{1}{2} \omega(2 t)\right] d t=\frac{1}{2(\gamma+1)} \int_{0}^{+\infty} t^{\gamma-1} \omega(2 t) d t
$$

which is positive.

Proposition 5.3. For every $\delta \in[1 / 2,1]$, there exists an infinite sequence of exchangeable 0-1 random variables $\left(X_{k}\right)_{k \geq 1}$ such that, if $\theta$ has the limiting distribution of $\bar{X}_{n}$,

$$
d_{W}\left(\bar{X}_{n}, \theta\right) \cong \frac{1}{n^{\delta}} .
$$

Conversly, if an infinite sequence of exchangeable 0-1 random variables $\left(X_{k}\right)_{k \geq 1}$ verifies (5.2) for some random variable $\theta$ and some $\delta>0$, then $\delta \in[1 / 2,1]$.

Proof: If $\delta \in(1 / 2,1)$ the existence of the sequence is insured by Propositions 5.1 and 5.2 (just take $\gamma=2 \delta-1$ ).

If $\delta=1$, from Theorem 1.2 it suffices to choose $\theta$ with a Beta distribution.

If $\delta=1 / 2$, taking $\theta$ with distribution a Dirac mass, say, at $1 / 2$, it is easy to see from the very definition of the Wasserstein distance that

$$
d_{W}\left(\theta+\sqrt{\frac{\theta(1-\theta)}{n}} Z, \theta\right)=\frac{\mathbb{E}|Z|}{2 \sqrt{n}},
$$

and from Proposition 3.3, this implies $d_{W}\left(\bar{X}_{n}, \theta\right) \cong 1 / \sqrt{n}$.

The converse is a direct consequence of Proposition 3.1, since if (5.2) holds then the distribution of $\theta$ is the limiting distribution of $\bar{X}_{n}$. 


\section{Acknowledgements}

GP would like to thank Pietro Rigo and Antonio Lijoi for useful discussions. GM's research is supported by a Welcome Grant from the Université de Liège. GP acknowledges support of the project F1R-MTH-PUL-15STAR / STARS at Luxembourg University. YS acknowledges support from the IAP Research Network P7/06 of the Belgian State (Belgian Science Policy). The authors thank the two anonymous referees for their remarks and questions which helped improve the paper.

\section{References}

D. J. Aldous. Exchangeability and related topics. In École d'été de probabilités de Saint-Flour, XIII-1983, volume 1117 of Lecture Notes in Math., pages 1-198. Springer, Berlin (1985). MR883646.

T. Austin. On exchangeable random variables and the statistics of large graphs and hypergraphs. Probab. Surv. 5, 80-145 (2008). MR2426176.

J. R. Blum, H. Chernoff, M. Rosenblatt and H. Teicher. Central limit theorems for interchangeable processes. Canad. J. Math. 10, 222-229 (1958). MR0096298.

L. H. Y. Chen, L. Goldstein and Q.-M. Shao. Normal approximation by Stein's method. Probability and its Applications (New York). Springer, Heidelberg (2011). ISBN 978-3-642-15006-7. MR2732624.

B. De Finetti. Funzione caratteristica di un fenomeno aleatorio. In Atti del congresso Internazionale dei Matematici (Bologna, 3-10 settembre 1928), pages 179-190 (1929).

B. De Finetti. Funzione caratteristica di un fenomeno aleatorio. Atti Reale Accademia Nazionale dei Lincei, Mem. 4, 86-133 (1930).

B. De Finetti. Foresight: Its logical laws, its subjective sources (1937). Studies in subjective probability pages 55-118 (1980).

P. Diaconis and D. Freedman. Finite exchangeable sequences. Ann. Probab. 8 (4), 745-764 (1980). MR577313.

C. Döbler. Stein's method of exchangeable pairs for the beta distribution and generalizations. Electron. J. Probab. 20, no. 109, 34 (2015). MR3418541.

O. El-Dakkak and G. Peccati. Hoeffding decompositions and urn sequences. Ann. Probab. 36 (6), 2280-2310 (2008). MR2478683.

O. El-Dakkak, G. Peccati and I. Prünster. Exchangeable Hoeffding decompositions over finite sets: a combinatorial characterization and counterexamples. J. Multivariate Anal. 131, 51-64 (2014). MR3252635.

A. L. Gibbs and F. E. Su. On choosing and bounding probability metrics. International statistical review $\mathbf{7 0}$ (3), 419-435 (2002). DOI: 10.1111/j.17515823.2002.tb00178.x.

L. Goldstein and G. Reinert. Stein's method for the beta distribution and the Pólya-Eggenberger urn. J. Appl. Probab. 50 (4), 1187-1205 (2013). MR3161381.

D. L. Hanson and L. H. Koopmans. Convergence rates for the law of large numbers for linear combinations of exchangeable and *-mixing stochastic processes. Ann. Math. Statist. 36, 1840-1852 (1965). MR0185643.

M. Hauray and S. Mischler. On Kac's chaos and related problems. J. Funct. Anal. 266 (10), 6055-6157 (2014). MR3188710.

E. Hewitt and L. J. Savage. Symmetric measures on Cartesian products. Trans. Amer. Math. Soc. 80, 470-501 (1955). MR0076206. 
B. M. Hill, D. Lane and W. Sudderth. Exchangeable urn processes. Ann. Probab. 15 (4), 1586-1592 (1987). MR905350.

H. Inoue and R. L. Taylor. Laws of large numbers for exchangeable random sets in Kuratowski-Mosco sense. Stoch. Anal. Appl. 24 (2), 263-275 (2006). MR2204712.

O. Kallenberg. Probabilistic symmetries and invariance principles. Probability and its Applications (New York). Springer, New York (2005). ISBN 978-0387-25115-8; 0-387-25115-4. MR2161313.

M. Klass and H. Teicher. The central limit theorem for exchangeable random variables without moments. Ann. Probab. 15 (1), 138-153 (1987). MR877594.

A. Kufner. Weighted Sobolev spaces. A Wiley-Interscience Publication. John Wiley \& Sons, Inc., New York (1985). ISBN 0-471-90367-1. MR802206.

G. Peccati. Hoeffding-ANOVA decompositions for symmetric statistics of exchangeable observations. Ann. Probab. 32 (3A), 1796-1829 (2004). MR2073178.

J. Pitman. Some developments of the Blackwell-MacQueen urn scheme. In Statistics, probability and game theory, volume 30 of IMS Lecture Notes Monogr. Ser., pages 245-267. Inst. Math. Statist., Hayward, CA (1996). MR1481784.

G. Stoica. Complete convergence of exchangeable sequences. Journal of Mathematics and Statistics 7, 95-98 (2011). DOI: 10.3844/jmssp.2011.95.97.

R. L. Taylor, P. Z. Daffer and R. F. Patterson. Limit theorems for sums of exchangeable random variables. Rowman \& Allanheld Probability and Statistics Series. Rowman \& Allanheld, Publishers, Totowa, NJ (1985). ISBN 0-8476-7435-5. MR860208.

R. L. Taylor and T. C. Hu. On laws of large numbers for exchangeable random variables. Stochastic Anal. Appl. 5 (3), 323-334 (1987). MR901022.

S. S. Vallender. Calculation of the wasserstein distance between probability distributions on the line. Theory of Prob. Appl. 18 (4), 784-786 (1974). DOI: $10.1137 / 1118101$.

C. Villani. Topics in optimal transportation, volume 58 of Graduate Studies in Mathematics. American Mathematical Society, Providence, RI (2003). ISBN 0-8218-3312-X. MR1964483.

N. C. Weber. A martingale approach to central limit theorems for exchangeable random variables. J. Appl. Probab. 17 (3), 662-673 (1980). MR580026. 